\title{
Prevalencia de hipertensión arterial y de sus factores de riesgo en estudiantes universitarios de Barranquilla, Colombia
}

\section{Prevalence of arterial hypertension and its risk factors in university students from Barranquilla, Colombia}

Olga Suárez Landazábal ${ }^{1}$, Carlos Villarreal Sotomayor ${ }^{2}$, Alexander Parody $^{3}$, Adel Rodríguez Delgado ${ }^{4}$, Roberto Rebolledo Cobos $^{5}$

\section{RESUMEN}

Introducción: La hipertensión arterial es un factor de riesgo cardiovascular muy prevalente en el mundo y una amenazaparaalcanzarunbuenestadodesalud.Objetivo:

\section{ABSTRACT}

Introduction: arterial hypertension, is a cardiovascular risk factor very prevalent in the world and, in turn, is an obstacle to the achievement of a good state of

\section{Historial del artículo:}

Fecha de recepción: 26/11/2018

Fecha de aprobación: 14/05/2019

1 Universidad Metropolitana de Barranquilla, Fisioterapeuta, Magíster en investigación y docencia universitaria. Programa de Fisioterapia, Barranquilla, Colombia.

2 Universidad Metropolitana de Barranquilla, Estudiante VIII semestre. Grupo semillero de investigación. Programa de Fisioterapia, Barranquilla, Colombia.

3 Universidad Metropolitana de Barranquilla, Ingeniero Industrial. Magister en Estadística Aplicada. Barranquilla, Colombia.

4 Universidad Autónoma del Caribe. Licenciado en Cultura Física y Deportes, Magíster en Actividad Física y Salud. Programa de Deportes y Cultura Física. Barranquilla, Colombia.

5 Universidad Metropolitana de Barranquilla, Fisioterapeuta, Magíster en Actividad Física y Salud. Programa de Fisioterapia. Barranquilla, Colombia.

Correspondencia: Olga Suárez Landazábal. Dirección: Calle 76 No 42-78 Barranquilla, Atlántico. Teléfono: 3008084648. Correo Electrónico: olga.suarez@unimetro.edu.co olgasula@gmail.com

Como citar este artículo: Suárez-Landazábal 0, Villarreal-Sotomayor C, Parody A, Rodríguez-Delgado A, Rebolledo-Cobos R. Prevalencia de hipertensión arterial y sus factores de riesgo en estudiantes universitarios de Barranquilla, Colombia. Revista de la Facultad de Ciencias de la Salud de la Universidad del Cauca. 2019; 21 (2) 16 - 23 
Determinar la prevalencia de hipertensión arterial y de los factores de riesgo asociados, en estudiantes de pregrado de una institución de educación superior de la ciudad de Barranquilla, Colombia, específicamente los relacionados con la inactividad física, la obesidad y los antecedentes familiares. Métodos: Estudio descriptivo transversal. La muestra fue de 260 estudiantes de los diferentes programas de pregrado. Resultados: El estudio mostró una prevalencia de hipertensión de $4.6 \%$ yde $9.2 \%$ de pre-hipertensión con predominio del sexo masculino. La prevalencia de los factores de riesgo estudiados fue la siguiente: antecedentes familiares con un $72.7 \%$ con mayor frecuencia de parte del padre (predominando la hipertensión arterial); obesidadabdominal con un $26.5 \%$ de predominio masculino. El 26.1\% fueron clasificados físicamente como insuficientemente activos y el $24.2 \%$ como inactivos con un predominio del sexo femenino en estos niveles. Conclusiones: Es esta población juvenil, la prevalencia de HTA es $4.6 \%$ y es predominante en el sexo masculino. Medidas tempranas deberían intervenir los factores modificables prevalentes.

Palabras claves: hipertensión; actividad física; factor de riesgo. (DeCS)

\section{INTRODUCCIÓN}

La hipertensión arterial (HTA) es una enfermedad crónica no transmisible que constituye un problema de salud pública, así como un gran obstáculo para el logro de un buen estado de salud en poblaciones adultas. Impone una gran carga económica y social (1). La HTA es considerada la principal causa de mortalidad en todo el mundo (2), con más de nueve millones de muertes anuales. Cuatro de cada diez adultos la padecen (3).

En América Latina y el Caribe, entre el 20 y 35\% de la población adulta presenta HTA y muchos desconocen su condición de hipertensos (4). En Bogotá, según los resultados del estudio CARMELA la prevalencia es de $13.4 \%$. Este estudio fue realizado en siete ciudades latinoamericanas incluida Bogotá. La prevalencia global de HTA en el estudio fue de $16.3 \%$ (5).

Por otra parte, si se analizan los factores de riesgo de la HTA, estos han venido en continuo aumento. En el caso de la obesidad, casi se ha triplicado en todo el mundo desde 1975 según la Organización Mundial de la Salud (OMS). En 2016, el 39\% de las personas adultas de 18 o más ańos tenían sobrepeso y el 13\% eran obesas (6). En Colombia, la health. Objective: to determine the prevalence of arterial hypertension and the associated risk factors in undergraduate students of a Higher Education Institution of the city of Barranquilla (Colombia), specifically those related to physical inactivity, obesity and family history. Method: Cross-sectional descriptive study. The representative sample was 260 students of the different undergraduate programs. Results: the study showed a prevalence of hypertension of $4.6 \%$ and $9.2 \%$ of pre-hypertension with predominance of the male sex. The prevalence of the risk factors studied was as follows: family history with $72.7 \%$ more frequently from the father, predominantly with a history of hypertension; abdominal obesity with a $26.5 \%$ male predominance; and in relation to physical inactivity, 26.1\% of the population classified as insufficiently active and $24.2 \%$ inactive, with a predominance offemales at these levels. Conclusions: In is this young population, the prevalence of arterial hypertension is $4.6 \%$ and is predominant in the male sex. Early measures should intervene the prevailing modifiable factors.

Keywords: hypertension; physical activity; risk factor. (MeSH)

Encuesta Nacional de la Situación Nutricional en Colombia (ENSIN) de 2015, encontró que uno de cada tres jóvenes y adultos tiene sobrepeso (37.7\%), mientras que uno de cada cinco es obeso $(18.7 \%)(7)$.

De forma paralela, el sedentarismo se convierte en otro factor trascendental, considerado como una verdadera epidemia y un serio problema de salud pública mundial (2, 8,9 ). La OMS expresa que al menos un $60 \%$ de la población mundial no realiza la actividad física necesaria para tener beneficios a la salud (9). Esta organización asegura que la inactividad física es el cuarto factor de riesgo de mortalidad más importante y el principal factor etiológico en el $30 \%$ de la carga mundial de cardiopatía isquémica (8).

Diferentes estudios han concluido que la carga genética es un factor importante en la adquisición de la HTA $(10,11)$. Los nińos de familias hipertensas tienden a tener valores de presión arterial mayores que los nińos de familias sin esta condición; además de presentar alteraciones estructurales y funcionales en sus arterias de manera precoz, que los pondría en riesgo de desarrollar esta patología $(12,13)$; fenómeno que no se aprecia en las relaciones entre la presión arterial de padres con hijos adoptivos (10). 
Existe una relación directa entre los valores de presión arterial en las primeras tres décadas de la vida, con el eventual desarrollo de HTA en un adulto maduro. Los cambios vasculares comienzan de forma temprana, silente y asintomática, aumentado la presencia de otras enfermedades cerebrovasculares (ECV) (14, 15). En la actualidad, hay más evidencia disponible que apoya que el origen de la HTA se encuentra en la juventud.

Por ello, constituye un imperativo realizar investigaciones que permitan detectar desde edades tempranas de la vida, aquellos factores que puedan ser determinantes en el desarrollo de enfermedades crónicas como la HTA. De ahíque la presente investigación planteó como objetivo determinar la prevalencia de la hipertensión arterial y de los factores de riesgo asociados, en estudiantes de una institución de educación superior de la ciudad de Barranquilla, Colombia, específicamente los relacionados con la inactividad física, la obesidad y los antecedentes familiares.

\section{METODOLOGÍA}

Se realizó un estudio descriptivo transversal, en 260 estudiantes de los diferentes programas de pregrado de la Universidad Metropolitana de la ciudad de Barranquilla, seleccionados por muestreo probabilístico estratificado por programa académico. Dicha institución educativa es de carácter privado forma profesionales en ciencias de la salud.

Los criterios de inclusión fueron ser estudiante activo de la Universidad, tener menos de 35 ańos de edad y posterior a la presentación del proyecto, aceptar participar por medio de la firma del consentimiento informado. Como criterio de exclusión se consideró la presunción o confirmación de embarazo y tener diagnóstico previo de hipertenso.

Las fuentes primarias de recolección fueron un cuestionario que tomó información sociodemográfica y el cuestionario GPAQ (Global Physical Activity Questionnaire) de la Organización Mundial de la Salud que mide niveles de actividad física.

Además se realizó el registro de la medición directa de la presión arterial y de las medidas antropométricas.

Para llevar a cabo la toma de datos y mediciones se entrenaron estudiantes de pregrado de la Universidad Metropolitana como auxiliares de investigación, labor que fue supervisada por los investigadores a cargo.

La toma de presión arterial, se realizó con el tensiómetro digital marca OMRON HEM-7114 calibrado, siguiendo las recomendaciones de la Sociedad Internacional de Hipertensión Arterial (16). El procedimiento se iniciaba solicitando al estudiante reposo durante cinco minutos antes de la toma de presión arterial. En los 30 minutos previos a la medición, el sujeto no debía haber ingerido alimentos, beber café o fumar. Se realizaban dos tomas de presión arterial, una en cada brazo, con una diferencia de cinco minutos y obtener el promedio de las dos medidas. Si entre la primera y la segunda medida existía una diferencia de $5 \mathrm{mmHg}$ o más, se realizaba una tercera medida y el promedio de las tres corresponderá al resultado final.

La presión arterial fue clasificada según el Séptimo Informe del Junta Nacional del Comité de Prevención, Detección, Evaluación y Tratamiento de la Hipertensión Arterial de la Organización Panamericana de la Salud, que establece que la presión arterial es normal con valores $<120 / 80 \mathrm{mmHg}$, Prehipertenso entre 120/80 y 139/89 mmHg, Hipertenso estadio I entre $140 / 90$ y $159 / 99 \mathrm{mmHg}$ e Hipertenso estadio II mayor de 160/100 mmHg (17).

La toma del peso se realizó en una báscula digital marca Tanita. Para tallar la población se utilizó un tallímetro marca SECA. Para la toma del perímetro abdominal y circunferencia de cadera se utilizó una cinta antropométrica marcaSECA. Los parámetros tomados para Colombiafueron los referentes de la Federación Internacional de Diabetes (IDF) para poblaciones de América Central y América del Sur que utiliza como definición de obesidad abdominal la presencia de un perímetro $\geq 90$ centímetros en hombres, $\geq 80$ centímetros en mujeres (18). El índice cintura/cadera: se calculó dividiendo el perímetro de la cintura en cm por el perímetro de la cadera en $\mathrm{cm}$. Se consideran normales valores $<0,8$ en mujeres y $<1$ en varones tal como lo establece la OMS. El índice de masa corporal (IMC): Según la OMS, es el peso en kilogramos dividido por la masa en metros cuadrados (IMC = peso en $\mathrm{Kg} / \mathrm{talla}^{2}$ en metros). Un IMC por debajo de 18.5 se considera bajo peso, entre $18.5 \mathrm{y}$ 24.9 es adecuado, entre 25 y 29.9 sobrepeso, y por encima de esa cifra hablamos de obesidad (19).

Para la variable niveles de actividad física (AF) se hizo a través del Cuestionario GPAQ (Global Physical Activity Questionnaire) de la OMS (20). El GPAQ tiene 16 preguntas en tres dominios (trabajo, transporte, recreación) y es usado en edades de 16-84 ańos. En el momento de la aplicación se realizó previamente una capacitación mediante diapositivas a los estudiantes incluidos en el estudio, para asegurar la respuesta indicada para cada ítem. Teniendo en cuenta el gasto energético y el tiempo empleado en la actividad física, sumando la actividad física realizada en todos los dominios 
del cuestionario, los estudiantes fueron clasificados como "Suficientemente Activos", "Insuficientemente Activos" e "Inactivos" de acuerdo a los siguientes criterios: suficientemente activo es un estudiante que durante una semana acumula 150 minutos de actividad física moderada, 075 minutos de actividad física vigorosa; insuficientemente activo: un estudiante que durante una semana reporta algo de actividad realizada pero no es suficiente para alcanzar los criterios de Suficientemente Activo; e Inactivo: un estudiante que durante una semana no realiza ningún tipo de actividad física, teniendo presente que el concepto de actividad física según la OMS es "cualquier movimiento corporal producido por los músculos esqueléticos con el consiguiente consumo de energía".

\section{Análisis Estadístico}

Se organizó la información a partir de la elaboración de una base de datos en Excel y los resultados estadísticos se generaron con del software estadístico Statgraphics versión 16. Las variables cuantitativas continuas como por ejemplo la edad y el IMC se clasificaron en grupos acorde a los valores obtenidos (edad) 0 a clasificaciones mundialmente reconocidas (IMC), convirtiéndose así en variables cualitativas ordinales, lo cual permitió que a todas las variables de estudio (sexo, edad, estado civil, presión arterial, estado nutricional, perímetro abdominal, nivel de actividad física y antecedentes familiares) se les establecieron frecuencias tanto absolutas como relativas, y se realizaron análisis entre la variable presión arterial (la variable de estudio) y los factores: sexo, estado nutricional, perímetro abdominal, nivel de actividad física y antecedentes de padre y/o madre, en todos los casos acompańados con el valor $P$ de la prueba chi-cuadrado correspondiente.

\section{Consideraciones éticas}

Para la realización de la investigación se tuvieron en cuenta los principios de la declaración de Helsinki y la resolución 8430 de Colombia (21), según la cual el estudio corresponde a una investigación sin riesgo. Siguiendo estos lineamientos la investigación contó con el aval del comité de Bioética (Acta de aprobación $\mathrm{N}^{\circ}$ 005/2015) de la Universidad Metropolitana. Este artículo se estructuró siguiendo las recomendaciones STROBE $(22,23)$.

\section{RESULTADOS}

Se evaluaron 260 estudiantes y su representación porcentual en los once programas académicos se observa en la tabla 1.
Se encontró un predominio de población femenina con un 75.8\%. La mayoría de la población era soltera (92.3\%), de estrato medio (60.0\%) y con edades entre 19-21 ańos (39.2\%). Los resultados de la caracterización sociodemográfica de los sujetos objeto de estudio se puede apreciar en la Tabla 2.

El $86.2 \%$ del total de los sujetos evaluados presentaron valores normales de presión arterial, encontrando el 9.2\% en estado pre hipertensivo y el $4.6 \%$ fueron hipertensos

Tabla 1. Distribución de la población según los programas académicos de la institución estudiada.

\begin{tabular}{lcc}
\hline \multicolumn{1}{c}{ Programa } & $\begin{array}{c}\text { Frecuencia } \\
\text { Absoluta(n) }\end{array}$ & $\begin{array}{c}\text { Frecuencia } \\
\text { Relativa (\%) }\end{array}$ \\
\hline Medicina & 112 & 43.08 \\
Enfermería & 46 & 17.69 \\
Nutrición & 28 & 10.77 \\
Odontología & 24 & 9.23 \\
Psicología & 18 & 6.92 \\
Optometría & 9 & 3.46 \\
Fisioterapia & 7 & 2.69 \\
Terapia Ocupacional & 6 & 2.31 \\
Fonoaudiología & 5 & 1.92 \\
Trabajo social & 3 & 1.15 \\
Bacteriología & 2 & 0.77 \\
\hline Total & 260 & 100 \\
\hline
\end{tabular}

Tabla 2. Características generales de la población estudiada.

\begin{tabular}{lcc}
\hline \multicolumn{1}{c}{ Variables } & $\begin{array}{c}\text { Frecuencia } \\
\text { Absoluta (n) }\end{array}$ & $\begin{array}{c}\text { Frecuencia } \\
\text { Relativa (\%) }\end{array}$ \\
\hline Hombres & Sexo \\
Mujeres & 63 & 24.2 \\
\hline \multicolumn{3}{c}{ Edad } \\
\hline $16-18$ & 92 & 75.8 \\
$19-21$ & 102 & 35.4 \\
$\geq 22$ & 66 & 39.2 \\
\hline \multicolumn{3}{c}{25.4} \\
\hline Soltero & Estado civil \\
Unión libre & 240 & 92.3 \\
Casado & 12 & 4.6 \\
\hline & 8 & 3.1 \\
\hline Alto & Estrato social \\
Medio & 44 & 16.9 \\
Bajo & 156 & 60.0 \\
\hline
\end{tabular}


con diagnóstico clínico, predominando en estas dos últimas categorías el sexo masculino. Los resultados del estado nutricional (IMC) de la población estudiada, revelan que el $26.6 \%$ de la población tiene sobrepeso y un 5.8\% presenta obesidad con mayor proporción de mujeres. En relación a los niveles de actividad física (AF) se encontró que el 49.6\% de los estudiantes son suficientemente activos, el 26.2\% son insuficientemente activos y el $24.2 \%$ son inactivos, con mayor proporción del sexo femenino al referirnos a estas categorías. En la tabla 3 se muestran las proporciones de los factores de riesgo cardiovascular identificados en hombres y mujeres, incluyendo los antecedentes familiares.

Al analizar el estado de tensión arterial con los diferentes factores de riesgo (tabla 4), se encontraron diferencias importantes en las variables: sexo masculino $(\mathrm{p}=0.000)$, estado nutricional $(\mathrm{p}=0.032)$ y obesidad abdominal $(\mathrm{p}=0.000)$.

Tabla 3. Distribución de factores de riesgo cardiovascular según el sexo.

\begin{tabular}{|c|c|c|c|c|c|c|}
\hline \multirow[t]{2}{*}{ Variables } & \multicolumn{2}{|c|}{$\begin{array}{c}\text { Hombres } \\
(n=63)\end{array}$} & \multicolumn{2}{|c|}{$\begin{array}{l}\text { Mujeres } \\
(\mathbf{n}=197)\end{array}$} & \multicolumn{2}{|c|}{$\begin{array}{c}\text { Total } \\
(\mathrm{n}=\mathbf{2 6 0})\end{array}$} \\
\hline & $\mathbf{n}$ & $\%$ & $\mathbf{n}$ & $\%$ & $\mathbf{n}$ & $\%$ \\
\hline \multicolumn{7}{|c|}{ Presión arterial } \\
\hline Normal & 35 & 55.6 & 189 & 95.9 & 224 & 86.2 \\
\hline Pre-hipertenso & 19 & 30.2 & 5 & 2.5 & 24 & 9.2 \\
\hline Hipertenso & 9 & 14.3 & 3 & 1.5 & 12 & 4.6 \\
\hline \multicolumn{7}{|c|}{ Estado nutricional } \\
\hline Bajo peso & 1 & 2.6 & 16 & 8.1 & 17 & 6.5 \\
\hline Normal & 38 & 60.0 & 120 & 60.9 & 158 & 60.8 \\
\hline Sobrepeso & 17 & 26.3 & 52 & 26.4 & 69 & 26.5 \\
\hline Obesidad & 7 & 11.1 & 9 & 4.6 & 16 & 6.2 \\
\hline \multicolumn{7}{|c|}{ Perímetro abdominal } \\
\hline Normal & 31 & 49.0 & 160 & 81.2 & 191 & 73.5 \\
\hline Aumentado & 32 & 51.0 & 37 & 18.8 & 69 & 26.5 \\
\hline \multicolumn{7}{|c|}{ Nivel de actividad física } \\
\hline Inactivo & 5 & 7.9 & 58 & 29.4 & 63 & 24.2 \\
\hline $\begin{array}{l}\text { Insuficiente- } \\
\text { mente activo }\end{array}$ & 15 & 23.8 & 53 & 26.9 & 68 & 26.2 \\
\hline Activo & 43 & 68.3 & 86 & 43.7 & 129 & 49.6 \\
\hline \multicolumn{7}{|c|}{ Antecedentes familiares } \\
\hline $\begin{array}{l}\text { De parte } \\
\text { del padre }\end{array}$ & 29 & 43.0 & 74 & 37.6 & 103 & 39.6 \\
\hline $\begin{array}{l}\text { De parte } \\
\text { de madre }\end{array}$ & 22 & 34.9 & 64 & 32.5 & 86 & 33.1 \\
\hline Ninguno & 12 & 19.0 & 59 & 29.9 & 71 & 27.3 \\
\hline
\end{tabular}

\section{DISCUSIÓN}

Este estudio evidencia una situación preocupante y es la prevalencia de hipertensión arterial en jóvenes (4,6\%), que se corrobora con otras investigaciones como las realizadas en 2013 en Cuba (24), con una prevalencia del 6,3\% en 2401 participantes y en 2014 (25) con una prevalencia de $4,0 \%$. Estos estudios se han realizado mediante mediciones con tensiómetro identificando personas hipertensas y prehipertensas bajo los mismos parámetros de valores del presente estudio. En Colombia, investigaciones en la ciudad de Pasto en el 2011 y Bogotá 2016, reportan frecuencias similares (5\%) de sujetos con HTA $(26,27)$. En la ciudad de Medellín en 2016 encontraron una población mayor de universitarios hipertensos (12\%) pero sin asociación con el sexo. Nuestro estudio documentó una mayor proporción de sexo masculino entre los factores de riesgo de HTA (28).

Tabla 4. Distribución de estudiantes según Factores de riesgo asociados a la Hipertensión Arterial y valores de presión arterial

\begin{tabular}{|c|c|c|c|c|c|c|c|}
\hline \multirow[t]{2}{*}{ Variables } & \multicolumn{2}{|c|}{$\begin{array}{l}\text { Normal } \\
(n=224)\end{array}$} & \multicolumn{2}{|c|}{$\begin{array}{c}\text { Pre-hi- } \\
\text { pertenso } \\
(n=24)\end{array}$} & \multicolumn{2}{|c|}{$\begin{array}{c}\text { Hiper- } \\
\text { tenso } \\
(n=12)\end{array}$} & \multirow[t]{2}{*}{$\begin{array}{c}\mathbf{P} \\
\text { valor }\end{array}$} \\
\hline & $\mathbf{n}$ & $\%$ & n & $\%$ & n & $\%$ & \\
\hline \multicolumn{8}{|c|}{ Sexo } \\
\hline Femenino & 189 & 95.94 & 5 & 2.54 & 3 & 1.52 & \multirow{2}{*}{0.0000} \\
\hline Masculino & 35 & 55.56 & 19 & 30.16 & 9 & 14.29 & \\
\hline \multicolumn{8}{|c|}{ Estado nutricional (IMC) } \\
\hline Bajo peso & 17 & 100 & 0 & 0 & 0 & 0 & \multirow{4}{*}{0.0321} \\
\hline Normal & 141 & 89.24 & 11 & 6.96 & 6 & 3.80 & \\
\hline Sobrepeso & 55 & 79.71 & 9 & 13.04 & 5 & 7.25 & \\
\hline Obesidad & 11 & 68.75 & 4 & 25 & 1 & 6.25 & \\
\hline \multicolumn{8}{|c|}{ Perímetro abdominal } \\
\hline Normal & 176 & 92.15 & 10 & 5.24 & 5 & 2.62 & \multirow{2}{*}{0.0000} \\
\hline Aumentado & 48 & 69.57 & 14 & 20.29 & 7 & 10.14 & \\
\hline \multicolumn{8}{|c|}{ Nivel de actividad física } \\
\hline Inactivo & 58 & 92.06 & 3 & 4.76 & 2 & 3.17 & \multirow{3}{*}{0.1538} \\
\hline $\begin{array}{l}\text { Insuficiente- } \\
\text { mente activo }\end{array}$ & 62 & 91.18 & 4 & 5.88 & 2 & 2.94 & \\
\hline Activo & 104 & 80.62 & 17 & 13.18 & 8 & 6.20 & \\
\hline \multicolumn{8}{|c|}{ Antecedentes familiares } \\
\hline $\begin{array}{l}\text { De parte del } \\
\text { padre }\end{array}$ & 86 & 83.50 & 13 & 12.62 & 4 & 3.88 & 0.1252 \\
\hline $\begin{array}{l}\text { De parte de } \\
\text { madre }\end{array}$ & 71 & 82.56 & 11 & 12.79 & 4 & 4.65 & 0.0883 \\
\hline $\begin{array}{l}\text { Del padre } y / o \\
\text { madre }\end{array}$ & 124 & 83.22 & 19 & 12.75 & 6 & 4.03 & 0.0525 \\
\hline
\end{tabular}


La HTA se encuentra como antecedente familiar de parte de los padres y se compara con resultados similares a los de otros autores $(12,26,29)$, quienes encontraron, respectivamente $53.0 \%, 45.8 \%$ y $40.3 \%$ de la población estudiada. En este último estudio, a diferencia del nuestro, se encontró una mayor proporción del antecedente por parte de la madre como factor de riesgo para HTA. Estos resultados son relevantes, considerando que, según la OMS, la HTA es uno de los principales factores de riesgo de enfermedad cardiovascular (7) incluyendo el infarto agudo de miocardio (30).

En cuanto al IMC, el estudio reveló sobrepeso y obesidad de predominio femenino al igual que el estudio de Hernández et al. (2010), con sobrepeso (29\%) y obesidad (5\%)(31); mientras que Almonacid et al., encontraron cifras menores de sobrepeso, $15.5 \%$ y $2.2 \%$ de obesidad (27). Datos similares a los de Arias et al., en 2007 donde mostró cifras superiores de sobrepeso (33.2\%) y de obesidad (16.7\%) en adolescentes de Medellín (32).

Con relación a la obesidad abdominal, los resultados del presente estudio están en consonancia con otros autores (24, 28). En nuestro estudio la obesidad abdominal en los hombres estaba presente de forma más importante en sujetos con la HTA.

Finalmente, de acuerdo a los hallazgos del nivel de actividad física, al menos el $50 \%$ de los estudiantes están por debajo de los requerimientos mínimos recomendados por la OMS. Esta situación es similar a la encontrada en 2009 por Lema et al. (33), con un 77\%; Ramos et al., con un 90\% de estudiantes sedentarios (26); Cardona et al. con un 66\% con baja actividad física (28). Además los estudios de Almonacid et al. (27), con un $63.8 \%$ y Hernández et al. (31), con $64.1 \%$ de inactivos físicamente, coinciden con los resultados de nuestro estudio, donde las mujeres son más inactivas que los hombres.

Hay que destacar que las investigaciones referenciadas utilizaron el Cuestionario Internacional de Actividad Física (IPAQ) $(27,28,31)$, cuestionarios y encuesta de elaboración propia, mientras que en nuestro estudio se utilizó el GPAQ. Consideramos que, aunque el IPAQ y GPAQ valoran frecuencia, duración e intensidad de la AF en dominios muy similares, el GPAQ tiene una ventaja frente al IPAQ, debido a que el GPAQ pregunta por la cantidad de actividad física en una semana "típica" (lo que regularmente se practica), mientras el IPAQ requiere que se establezcan solo la actividad física realizada en los últimos siete días.

Como limitaciones del estudio se encuentra el sesgo de memoria, al preguntar por aspectos tales como antecedentes familiares, y que las asociaciones estadísticas no son causales sino exploratorias pero que a la vez se constituyen en base para formulación de hipótesis. El análisis de este estudio no incluyó ninguna forma de ajuste y una de sus limitaciones es la ausencia de control de efectos confusores en las asociaciones documentadas, las cuales fueron crudas.

De acuerdo a lo expuesto el panorama es preocupante y desalentador considerando que se trata de una población joven que, de no cambiar su estilo de vida, la probabilidad de eventos cardiovasculares será mayor, afectando no solo al individuo sino a la sociedad. Por tanto, las instituciones educativas en general deben implementar cursos obligatorios y electivos basados en la práctica de estilos de vida saludables, fomentar la práctica de ejercicio físico o deporte en los tiempos libresy ofertar alimentos saludables a costos asequibles (34).

\section{CONTRIBUCIÓN DE LOS AUTORES, CONFLICTOS DE INTERÉS Y FUENTES DE FINANCIAMIENTO}

OSL. Concepción del proyecto original, planificación, obtención de datos, interpretación de los resultados y redacción inicial del manuscrito.

CVS. Obtención de datos, interpretación de resultados.

AP. Interpretación estadística de los resultados, redacción de la metodología y de resultados en el manuscrito.

ARD Interpretación de resultados, redacción final del manuscrito.

RRC. Interpretación de resultados, redacción final del manuscrito.

Los autores declaramos que no existe conflicto de interés para la publicación del presente artículo. Este artículo es producto del trabajo realizado por el grupo de investigación "Cuidado de la salud y la vida", de la Universidad Metropolitana de la ciudad de Barranquilla, Colombia.

Para la realización del presente trabajo de investigación se contó con el apoyo económico de la Universidad Metropolitana de Barranquilla.

\section{REFERENCIAS}

1. Sánchez R, Ayala M, Baglivo H, Velázquez C, Burlando G, Kohlmann 0, et. al. Guías Latinoamericanas de Hipertensión Arterial. Rev Chil Cardiol. 2010; 29(1): 117-44. 
2. Castro J, Abellán J, Leal M, GómezP, Ortín E, Abellán Alemán $\mathrm{J}$. Lifestyles related with cardiovascular risk in university students. Clin Investig Arterioscler. 2014; 26(1):10-6.

3. Delgado P, Alarón M, Caamańo F. Analysis of cardiovascular risk factors in young university students according to their nutritional status. Nutr Hosp. 2015; 32(4):1820-4.

4. Ruilope L, Chagas A, Brandăo A, Gómez-Berroterán R, Alcalá J, Paris J, Cerda J. Hypertension in Latin America: Current perspectives on trends and characteristics. Hipertens Riesgo Vasc. 2017; 34(1):50-6.

5. Silva H, Hernandez-Hernandez R, Vinueza $R$, et al. CARMELA Study Investigators. Cardiovascular risk awareness, treatment, and control in urban Latin America. Am J Ther. 2010; 17: 159-66.

6. NCD Risk Factor Collaboration. Worldwide trends in bodymass index, underweight, overweight, and obesity from 1975 to 2016: a pooled analysis of 2416 population-based measurement studies in 128.9 million children, adolescents, and adults. Lancet. 2017; 390(10113): 2627-42.

7. Encuesta Nacional de Situación Nutricional de Colombia (ENSIN) [Internet]. Ministerio de Salud (Colombia); 2015 [citado 10 de Enero de 2018]. Recuperado a partir de: http://cort.as/-Skry

8. Hills A, Street S, Byrne N. Physical Activity and Health: "What is Old is New Again". Adv Food Nutr Res. 2015; 75:77-95.

9. Budnik A, Henneberg M. Worldwide increase of obesity is related to the reduced opportunity for natural selection. PLoS One. 2017; 12(1): 23-9.

10. Abraham W, Blanco W; Coloma G, Cristaldi A, Gutiérrez N, Sureda L. Estudio de los factores de riesgo cardiovascular en adolescentes. Rev Fed Arg Cardiol. 2013; 42(1): 29-34.

11. Van der Sande W, Milligan B, Ceesay N. Factores de riesgo de la hipertensión arterial y la salud cardiovascular en estudiantes universitarios. 2006: Servicio de Publicaciones de la Universidad de Murcia. Murcia (Espańa).

12. Ingaramo R, Mitrano A, Cattaneo S. Valoración de la estructura y la función arterial en adolescentes normotensos hijos de padres hipertensos. Rev Fed Arg Cardiol 2009; 38(4): 200-6.

13. Cortés 0 , Grupo PrevInfad/PAPPS Infancia y Adolescencia. Prevención de la hipertensión arterial en la infancia y la adolescencia. Rev Pediatr Aten Primaria. 2008; 10(1): 669-97.

14. Lai C, Sun D, Cen R, Wang J, Li S, Fernandez-Alonso C, et al. Impact of Long-Term Burden of Excessive Adiposity and Elevated Blood Pressure From Childhood on Adulthood Left Ventricular Remodeling Patterns: The Bogalusa Heart Study. J Am Coll Cardiol. 2014; 64 (15): 1580-7.

15. Samuels J, Bell C, Samuel J, Swinford R. Management of Hypertension in Children and Adolescents. Curr Cardiol Rep. 2015; 17(12):107-12.
16. Ruilope L. Current challenges in the clinical management of hypertension. Nat Rev Cardiol. 2011; 9(5): 267-75.

17. Axon R, Turner M, Buckley R. An Update on Inpatient Hypertension Management. Curr Cardiol Rep. 2015; 17(11):94.

18. Medina J. Prevalencia de Sobrepeso y Obesidad en la Población Adulta de Arequipa Metropolitana: Resultados del Estudio PREVENCIÓN. Rev Per Cardiol. 2006; 32(3) 27-34.

19. World Health Organization. Obesity: Preventing and Managing the Global Epidemic. Report of a WHO Consultation of Obesity. Geneva, 2000.

20. Chu A, Ng S, Koh D, Müller-Riemenschneider F. Reliability and Validity of the Self- and InterviewerAdministered Versions of the Global Physical Activity Questionnaire (GPAQ). PLoS One. 2015; 10(9): 45-38.

21. Ministerio de Salud de Colombia. Resolución Número 8430 de 1993.

22. Bravo Peńa M, Barona Fong L, Campo López J, Calvache JA. El reporte de la investigación observacional y la declaración STROBE. Revista Facultad Ciencias de la Salud: Universidad del Cauca. 2014; 16(3):39-45.

23. Von Elm E, Altman DG, Egger M, Pocock SJ, Gřtzsche PC, Vandenbroucke JP. The Strengthening the Reporting of Observational Studies in Epidemiology (STROBE) statement: guidelines for reporting observational studies. Annals of internal medicine. 2007; 147(8):573-7.

24. Guerra E, Vázquez J, Dominica Y, Hinojosa Y, Chang A. Caracterización de pacientes con hipertensión arterial en el Policlínico Universitario "Ramón López Peńa". MEDISAN. 2013; 17(4): 599-604.

25. Rodríguez L, Díaz M, Ruiz V, Hernández H, Herrera V, Montero M. Factores de riesgo cardiovascular y su relación con la hipertensión arterial en adolescentes. Rev Cub Med. 2014; 53(1): 25-36.

26. RamosJ.Prevalencia dehipertensión y pre hipertensión en jóvenes. Rev Univ Salud. 2011; 2 (14): 68-78.

27. Almonacid C, Camarillo M, Murcia Z, Medina C, Rebellon J, Mendieta H. Evaluación de factores de riesgo asociados a enfermedad cardiovascular en jóvenes universitarios de la Localidad Santafé en Bogotá, Colombia. NOVA. 2016; 13(25); 35-45.

28. Cardona-Arias J. Prevalencia de hipertensión arterial en universitarios, Medellín. Curare. 2014; 1(1): 17-26.

29. Cruz-Sánchez A, Orosio-Méndez B, Cruz-Ramírez C, Bernardino-García D, Vásquez-Domínguez E, GalindoPalma F, et al. Factores de riesgo cardiovascular en estudiantes de enfermería de una universidad pública. Enfermería Universitaria. 2016; 13(4): 226-32.

30. Mesa-Melgarejo L. Galindo LM. Factores de riesgo en Infarto Agudo de Miocardio: revisión de estudios observacionales. 
Revista de la Facultad de Ciencias de la Salud de la Universidad Del Cauca, 2015; 17(3), 10-20.

31. Arias R, Agudelo G. Prevalencia de algunos componentes del síndrome metabólico en escolares y adolescentes con sobrepeso y obesidad. Hallazgos del estudio de factores de riesgo para enfermedad cardiovascular en escolares y adolescentes. Perspect Nut Hum. 2007; 9(2): 11-22.

32. Hernández J, Herazo Y, Valero M. Frecuencia de factores de riesgo asociados a enfermedades cardiovasculares en población universitaria joven. Rev Salud Pub. 2010; 12(3): 852-64.

33. Lema L, Salazar I, Varela M, Díaz J, Rubio A, Botero A. Comportamiento y salud de los jóvenes universitarios: Satisfacción con el estilo de vida. Pensamiento Psicológico. 2009; 5(12): 71-88.

34. García-Laguna D, García-Salamanca G, Tapiero-Paipa Y, Ramos D. Determinantes de los estilos de vida y su implicación en la salud de jóvenes universitarios. Rev Prom Salud. 2012; 17(2): 169-85. 